\title{
Primary thyroglossal duct carcinoma with lateral neck metastasis in an adolescent girl
}

\author{
Patricia Papendieck*, Laura Gruneiro-Papendieck and Ana Chiesa \\ Division of Endocrinology, Endocrinology Research Center, Dr. Cesar Bergadá (CEDIE) Children's Hospital "Ricardo Gutiérrez”, Rooster 1330, C1425EFD \\ Buenos Aires, Argentina
}

\begin{abstract}
Thyroglossal duct cysts are the most common thyroid developmental anomalies accounting for $75 \%$ of midline neck tumors in children. Carcinomas arising from this remnant are very rare in adults and even scarcer in pediatrics. Preoperative diagnosis is a challenge and appropriate treatment is controversial. We report the case of a 13-year-old adolescent girl with a growing midline neck mass suspicious for a thyroglossal duct cyst who underwent a Sistrunk procedure. Histologic analysis revealed the presence of a papillary carcinoma in the wall. She completed treatment with total thyroidectomy and radioactive iodine. Postablative whole body scan showed an inferior neck metastasis. We discuss previous pediatric cases of this entity and the different options in the management strategy.
\end{abstract}

\section{Introduction}

Thyroglossal duct cysts (TGDC) are the most common thyroid developmental anomalies accounting for $75 \%$ of midline neck tumors in children and approximately $7 \%$ in adults [1-3].

As the thyroid gland descends from the foramen caecum to its final location overlying the laryngeal prominence of the trachea, it leaves behind an epithelial tract known as thyroglossal duct that disappears by the 5-10th gestational week. Partial persistence of this duct creates the basis for the origin of a thyroglossal duct remnant that can be a cyst, a tract or duct or a fistula and can also harbor ectopic thyroid tissue (1-45\% of the cases) [1,3]. Carcinomas arising from this remnant are very rare in adults $(<1 \%)$ with approximately 250 reported cases in the literature since its first description by Brentano in 1911 [3,4]. So far, in pediatrics only 27 cases have been reported $[1,5]$.

The purpose of this paper is to describe a case of a pediatric primary thyroglossal duct carcinoma (TGDCa) with lateral neck metastasis.

\section{Case presentation}

A 12-year-old girl presented with an asymptomatic fast-growing neck mass noticed 7 months before consultation. Her previous medical history was unremarkable without past neck radiation. Neck ultrasound (US) revealed a cystic-solid mass of $21 \times 13 \mathrm{~mm}$ with microcalcifications in the upper cervical region near the hyoid bone with an eutopic and normal thyroid gland. With a presumptive diagnosis of TGD remnant a Sistrunk procedure was performed. Histological evaluation found a papillary carcinoma of $12 \times 6 \mathrm{~mm}$ in the wall of the TGD (Figure 1). The patient was referred to our unit for follow up.

Physical examination revealed an euthyroid postmenarcheal girl with a non-palpable thyroid gland without palpable cervical nodes. Thyroid function was normal: TSH $0.95 \mathrm{mU} / \mathrm{L}$ (RV: 0.5-5), T4 $9.1 \mathrm{ug} / \mathrm{dl}$ (RV: 6-16), FT4 $1.16 \mathrm{ng} / \mathrm{dl}$ (RV: 0.8-2) with negative thyroid antibodies. Neck US showed an eutopic thyroid gland with a small solid nodule $(5 \times 3 \mathrm{~mm})$ on the left lobe and normal cervical lymph nodes. Chest CT scan was normal. In order to complete her treatment, she underwent a total thyroidectomy. No suspicious adenopathies were found Pathological examination did not reveal thyroid tumor. Postoperative ${ }^{131} \mathrm{I}(50 \mathrm{mCi})$ was administered with TSH $60 \mathrm{mU} / \mathrm{L}$ and serum thyroglobulin (Tg) $47 \mathrm{ng} / \mathrm{ml}$. A whole body scan (WBS) performed on day 5 showed a focal uptake confined to the inferior cervical or superior mediastinal region (Figure 2). US evidenced a suprasternal adenopathy of $15 \times 7 \mathrm{~mm}$ with a heterogeneous vascularized rounded area. A US guided FNAB was positive for papillary carcinoma (washout Tg: $52400 \mathrm{ng} / \mathrm{ml}$ ). Surgical excision confirmed lymph node infiltration of papillary carcinoma. Four months later (TSH :100 mU/L, Tg $9.2 \mathrm{ng} /$ $\mathrm{ml}$ ) she received $100 \mathrm{mCi}{ }^{131} \mathrm{I}$ and $\mathrm{WBS}$ was negative. During followup suppressed $\mathrm{Tg}$ was negative but stimulated $\mathrm{Tg}$ (sTg) remained positive (between 10-20 ng/ml). WBS showed uptake in the anterior mediastinum but 18-FDG-PET only proved physiologic thymic uptake. At 5 years of diagnosis, she still has positive sTg (slowly decreasing levels) with persistent anterior mediastinal uptake (Table 1).

\section{Discussion}

We present the unusual case of an adolescent girl with a primary TGDCa and neck metastasis.

As described in the literature the initial clinical presentation was a simple thyroglossal duct cyst with the incidental diagnosis of papillary carcinoma on pathologic examination [1-4].

Preoperative diagnosis of TGDCa is challenging, some clinical or ultrasonographic signs can be suggestive of malignancy as

Correspondence to: Patricia Papendieck, Division of Endocrinology, Endocrinology Research Center, Dr. Cesar Bergadá (CEDIE) Children's Hospital "Ricardo Gutiérrez", Rooster 1330, C1425EFD Buenos Aires, Argentina, Tel: +541149635931; E-mail: ppapendieck@cedie.org.ar

Key words: thyroglossal duct cyst carcinoma, pediatric, neck metastasis

Received: February 11, 2018; Accepted: February 24, 2018; Published: February 27,2018 


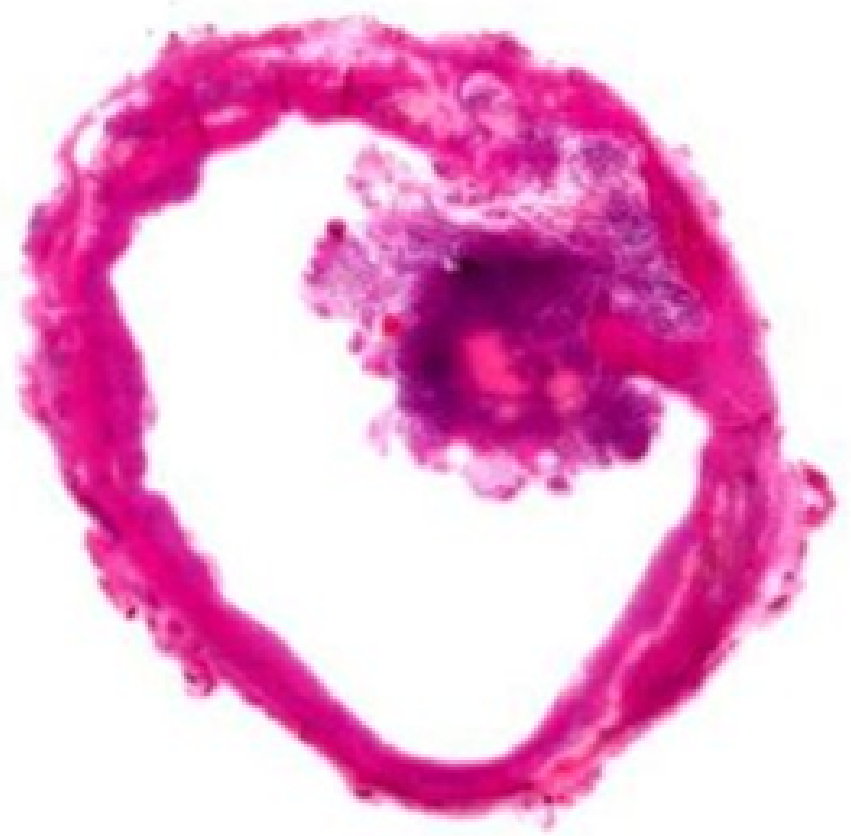

Figure 1. Macrocopy of TGD cyst with carcinoma in the wall.

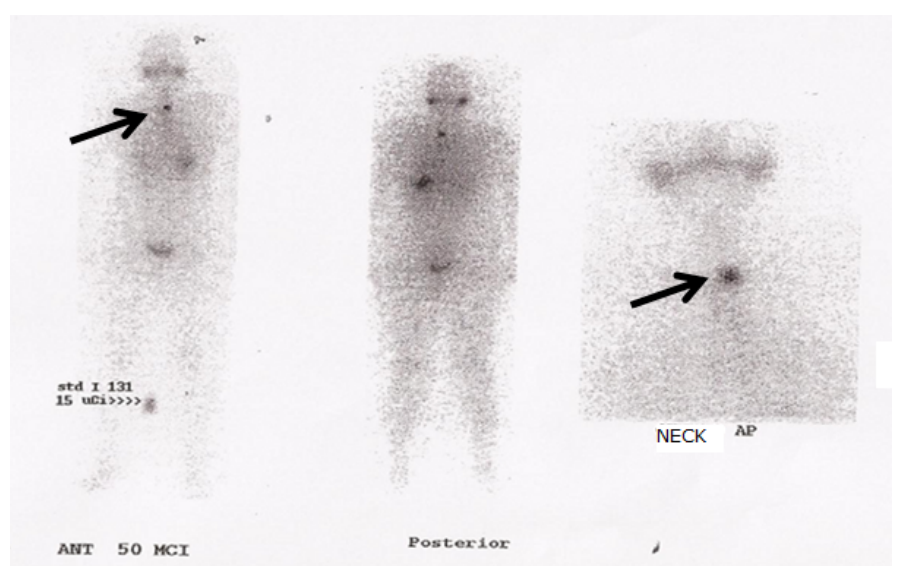

Figure 2. Postablative WBS: abnormal uptake confined to the inferior cervical or superior mediastinal region.

fixation to hyoid bone or surrounding structures, the presence of microcalcifications or fast growth in the absence of infection. Because the TGDCa is an infrequent condition clinician rarely suspect it and the diagnosis is often unexpected and made, as in our case, postoperatively $[1,3,4]$.

US appearance of TGDC is variable ranging from anechoic to heterogeneous complex cystic lesions with a pseudosolid appearance (1). The presence of a solid component in a TGD remnant should alert the physician and raises the possibility of a TGDCa $[1,3,4]$. If a FNAB is performed US guidance is required in order to sample the solid part of the lesion -usually the wall-. Few reported patients underwent FNAB preoperatively while most patients were referred for treatment after surgery with histological diagnosis of papillary TGDCa. Moreover, even with a suspected diagnosis, FNAB in them wasn't accurate due to hypocellularity [1].

Before the surgical remove of a thyroglossal remnant a normal located thyroid gland must be evidenced. The ectopic thyroid tissue contained in the cyst may be the only functioning thyroid tissue and its removal may render the patient hypothyroid [1]. When the thyroid gland is not demonstrated in place by the US, a preoperative scan may be useful to detect ectopic localization.

The common surgical treatment for benign or malignant TGD remnants in order to diminish the possibility of recurrence is the Sistrunk procedure $[1,3,4,6-8]$. It consists in the resection of the cyst, the body of hyoid bone and a cone of the base of tongue muscle up to the foramen caecum. Coinciding with all pediatric reported cases our patient harbored a papillary carcinoma [1].

The extension of the surgical procedure is a point of controversy reliant on the different concepts about the origin of the TGDCa. Some authors argue that these lesions arise de novo within the islands of ectopic thyroid tissue of the cyst (primary TGDCa) whereas others believe that the tumor is a metastasis from an occult carcinoma of the thyroid gland (synchronous TGDCa) [3]. Ectopic thyroid rests present in many of the TGD remnants obtained by surgery support the novo hypothesis, while the finding in adults of unsuspected TGDCa in papillary thyroid cancers who underwent surgery favors the metastasis theory [6,7].

Our patient, as all pediatric patients with TGDCa who underwent thyroidectomy, did not have concomitant thyroid cancer. In this regard, even when the thyroid tumor is found, prophylactic neck dissection is under controversy because of the tumor's low risk of recurrence $[1,3,4]$. Distant metastases are very rare in this entity and mainly described in adults $(1,3 \%)[6,7]$.

Regional lymph node metastases have been reported in $23 \%$ of pediatric patients with TGDCa [1]. Only one pediatric patient presented pulmonary metastases ${ }^{(1)}$. The finding in our patient of a suprasternal metastasis in region VII without thyroid involvement supports the primary TGDCa origin.

As in thyroid papillary carcinoma the prognosis is favorable and evidenced by the fact that only one reported patient died postoperatively and by surgery complications [1]. Papillary TGDCa is probably not different from thyroid papillary carcinoma which is frequently a multifocal disease. Therefore associating thyroidectomy to the Sistrunk procedure will facilitate surveillance of the disease with subsequent ${ }^{131} \mathrm{I}$ ablation and long term monitoring with $\mathrm{Tg}$ measurements [3,7]. In fact, with this strategy, our patient had a favorable outcome at 5 years of follow up with no structural disease and only biochemical persistence (low sTg levels).

\section{Conclusion}

Although exceptional in pediatrics, rapid growth of a TGD cyst in the absence of infection and/ or US signs suggestive of malignancy should alert the physician of the possibility of a TGDCa. The lack of thyroid involvement does not rule out the presence of metastasis and follow-up should be the same as for differentiated thyroid carcinoma. The more important advantage of total thyroidectomy is to allow ablative radioiodine therapy and follow-up with Tg measurements. As proposed by many other authors the treatment of these cancers should follow current guidelines for differentiated thyroid carcinoma [3,4].

\section{References}

1. Peretz A, Leiberman E, Kapelushnik J, Hershkovitz E (2004) Thyroglossal duct carcinoma in children: case presentation and review of the literature. Thyroid 14: 777785. [Crossref]

2. Hilger AW, Thompson SD, Smallman LA, Watkinson JC (1995) Papillary carcinoma arising in a thyroglossal duct cyst: a case report and literature review. J Laryngol Otol 109: 1124-1127. [Crossref] 
3. Storani ME, Vazquez AM, Alcaraz GN, Gutierrez S, GaunA, et al. (2010) Carcinoma of thyroglossal cyst. Argentine Journal of Endocrinology and Metabolism 47: 18-24.

4. Rayess HM, Monk I, Svider PF, Gupta A, Raza SN, et al. (2017) Thyroglossal Duct Cyst Carcinoma: A Systematic Review of Clinical Features and Outcomes. Otolaryngol Head Neck Surg 156: 794-802. [Crossref]

5. Pfeiffer MS, Kim GH, Krishnan M3 (2014) Thyroglossal duct papillary carcinoma in a 15 -year old female and review of pediatric cases of thyroglossal duct carcinoma. Int $J$ Pediatr Otorhinolaryngol 78: 135-138. [Crossref]
6. Patel SG, Escrig M, Shaha AR, Singh B, Shah JP (2002) Management of welldifferentiated thyroid carcinoma presenting within a thyroglossal duct cyst. J Surg Oncol 79: 134-139. [Crossref]

7. Pellegriti G, Lumera G, Malandrino P, Latina A, Masucci Ret al. (2013) Thyroid cancer in thyroglossal duct cysts requires a specific approach due to its unpredictable extension. J Clin Endocrinol Metab 98: 458-465. [Crossref]

8. Thompson LDR, Herrera HB, Lau SK (2017) Thyroglossal Duct Cyst Carcinomas in Pediatric Patients: Report of Two Cases with a Comprehensive Literature Review. Head Neck Pathol 11: 442-449. [Crossref]

Copyright: (C2018 Papendieck P. This is an open-access article distributed under the terms of the Creative Commons Attribution License, which permits unrestricted use, distribution, and reproduction in any medium, provided the original author and source are credited. 